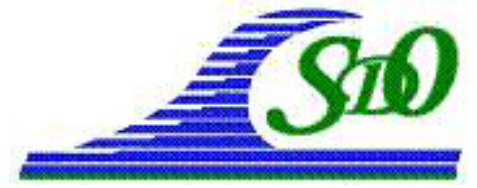

XI ìmes Journées Nationales Génie Côtier - Génie Civil

Les Sables d'Olonne, 22-25 juin 2010

DOI:10.5150/jngcgc.2010.003-B C Editions Paralia CFL

disponible en ligne - http://www.paralia.fr - available online

\title{
Modélisation couplée vagues-courant : développements avec MARS3D et WAVEWATCH III
}

\author{
Anne-Claire BENNIS ${ }^{1}$, Fabrice ARDHUIN ${ }^{2}$, Tina ODAKA ${ }^{3}$
}

1. Université Bordeaux I, UMR 5805EPOC, avenue des facultés, 33405 Talence, France. anne-claire.bennis@u-bordeaux1.fr

2. Ifremer, Laboratoire d'Océanographie Spatiale, BP 70, 29280 Plouzané, France. ardhuin@ifremer.fr

3. Ifremer, Ressources Informatiques et Communications, BP 70, 29280 Plouzané, France.

tina.odaka@ifremer.fr

\section{Résumé :}

Un modèle numérique couplant la circulation tridimensionnelle, résolue par le code MARS3D, et l'état de la mer, calculé par le code WAVEWATCH III, est développé pour étudier l'hydrodynamique des zones côtières et littorales. Le principe général du modèle, faisant appel au coupleur PALM est décrite, ainsi que les aménagements apportés aux deux codes existants afin de rendre le couplage possible. Des premiers tests en deux dimensions horizontales ont permis de développer une première version. En trois dimensions, nous montrons que le modèle doit calculer la vitesse quasiEulérienne, car il n'existe pas de forme analytique cohérente du forçage par les vagues pour la vitesse Lagrangienne. Cet aspect est illustré par un résultat comportant de très fortes erreurs par rapport à la solution analytique, obtenu avec le forçage erroné proposé par MELLOR (2003).

\section{Mots-clés :}

Modélisation couplée - MARS3D - WAVEWATCH III - Circulation côtière

\section{Introduction}

L'hydrodynamique littorale est le résultat d'interactions complexes entre états de mer, courants et niveaux d'eau. La compréhension de ces phénomènes hydrodynamiques est par ailleurs nécessaire à la gestion des zones côtières du fait de leur rôle dans le transport sédimentaire, le transport de larves et polluants, et le risque qu'ils représentent pour les activités nautiques. Si les phénomènes d'interaction entre l'état de la mer et des courants uniforme sur la verticale sont désormais assez bien connus, plusieurs effets observés semblent être intimement liés à la forte variation du courant sur la verticale, en particulier dans la zone de déferlement, avec le mélange horizontal induit par le cisaillement vertical (SVENDSEN \& PETREVU, 1994), ou la concentration en surface de courants opposés aux vagues (ARDHUIN, 2005), mais aussi sur le plateau 
continental avec la formation de sous-courants (LENTZ et al., 2008). Afin d'étudier ces phénomènes nous avons donc décider de développer un outil de modélisation numérique, d'abord à des fins de recherche. Cet outil s'appuie sur deux modèles matures et dont les méthodes numériques, peu diffusives, sont bien adaptées à l'étude de phénomènes présentant de fort gradients. Il s'agit des codes MARS3D (LAZURE \& DUMAS, 2008) pour la circulation océanique, et WAVEWATCH III (ci-dessous WWATCH) pour les états de mer (TOLMAN, 2008, ARDHUIN et al., 2009). Afin de minimiser les modification des deux codes et de permettre un calcul efficace sur un supercalculateur, le coupleur PALM (BUIS et al., 2008) a été utilisé.

\section{Principe du couplage et illustration en deux dimensions}

L'utilisation du coupleur PALM permet une plus grande souplesse dans les échanges entre les deux codes de calcul et permet une minimisation des modifications à effectuer dans les codes MARS3D et WWATCH. Lorsqu'il y a des nouvelles variables à calculer, toutes les opérations sont effectuées dans les subroutines intermédiaires wwtomars.f90 et marstoww.f90 (cf. figure 1) dans le but de rentrer dans les codes sources des deux modèles uniquement des variables prêtes à l'emploi. Il s'agit d'un couplage explicite dans lequel les informations sont échangées à chaque pas de temps ou non. Les grilles de calcul de MARS3D et WW3 sont pour l'instant les mêmes mais à terme il est prévu qu'elles soient différentes car MARS3D et WWATCH résolvent des processus qui mettent en jeu des échelles spatiales différentes.

Le couplage a tout d'abord été testé et validé dans un cas bidimensionnel (2DH). Une approche par tension de radiation (LONGUET-HIGGINS 1970a, 1970b) a été utilisée pour forcer le modèle de circulation par le modèle de vagues. La validation $2 \mathrm{DH}$ a été faite en zone de déferlement avec les données de l'expérience NSTS (THORNTON \& GUZA, 1986, WU et al., 1985). Il s'agit d'étudier la propagation d'une houle oblique avec une incidence de 20 degrés sur une plage plane (ie. sans barre) dont la bathymétrie diminue avec la distance cross-shore et de s'intéresser particulièrement au courant longshore généré par le déferlement. La comparaison avec les données in situ a été faite sur les profils de hauteur significative et de vitesse longshore en fonction de la distance cross-shore. Le code couplé reproduit bien l'intensité du pic de la vitesse longshore par contre la décroissance du courant après le pic est plus faible que celle observée in situ, ce qui est due à la paramétrisation de la viscosité horizontale qui pourrait être grandement améliorée (cf. figure 2). La hauteur significative simulée est du même ordre que celle observée in situ même si un léger décalage du point de déferlement est à noter. Le code couplé reproduit bien les phénomènes de set-up et de set-down observés in situ (cf. figure 3). Sur ce cas test, l'impact de la rétroaction a aussi été étudié et il s'avère que l'on constate des différences visibles entre les cas avec et sans rétroaction mais que celles-ci restent faibles. 


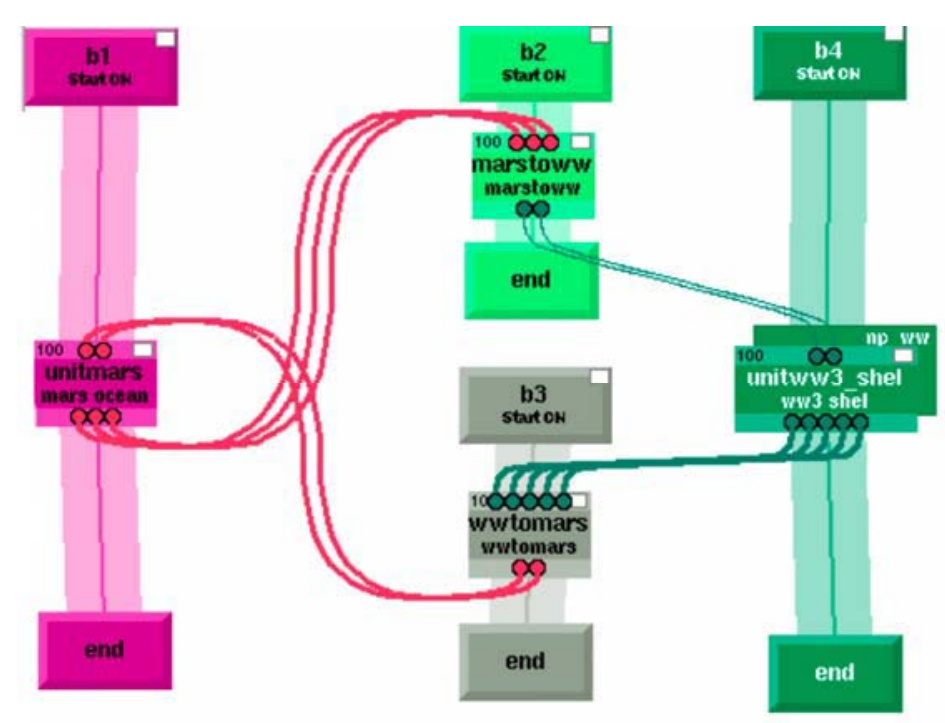

Figure 1. Exemple de canevas prepalm.
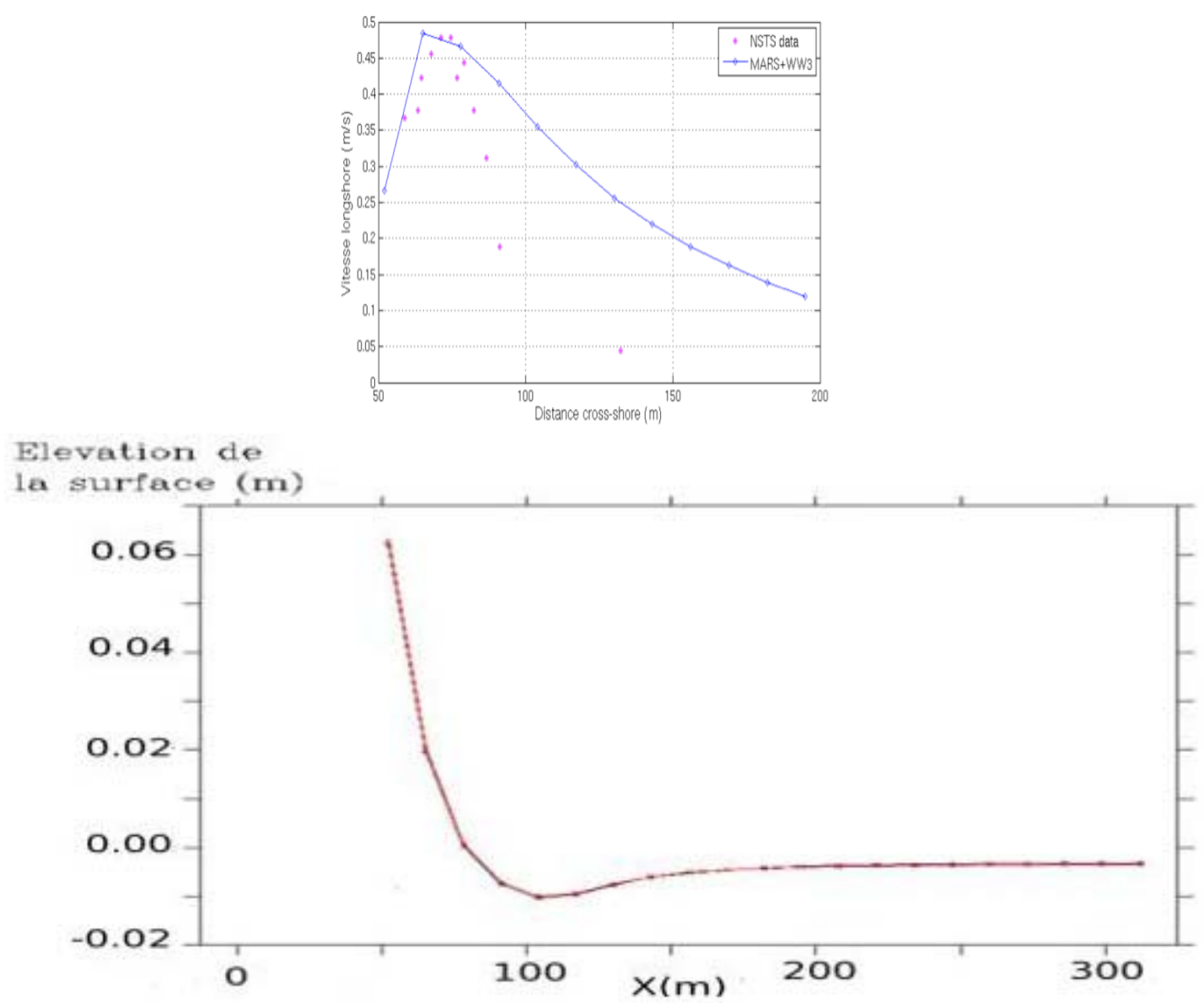

Figure 2. En haut : comparaison de vitesses longshore entre données modèle (trait continu) et données in situ (points). En bas : comparaison de l'élévation de la surface entre le cas couplé (en rouge) et le cas forcé (en noir). 


\section{Modèle couplé en trois dimensions}

Le premier problème qui se pose en trois dimensions est le choix des équations de base pour l'évolution de la quantité de mouvement horizontale. Soit on écrit l'évolution de la vitesse Lagrangienne moyenne $U$ qui comprend la dérive de Stokes $U_{s}$ associée aux vagues, soit on écrit l'évolution de la vitesse quasi-Eulérienne définie à peu près comme $u^{\prime}=U-U_{S}(\mathrm{ARDHUIN}$ et al., 2008a). Dans ce dernier cas on obtient,

$$
\begin{aligned}
& \frac{\partial u^{\prime}}{\partial t}+u^{\prime} \frac{\partial u^{\prime}}{\partial x}+v^{\prime} \frac{\partial u^{\prime}}{\partial y}+w^{\prime} \frac{\partial u^{\prime}}{\partial z}-f v^{\prime}+\frac{1}{\rho} \frac{\partial p^{H}}{\partial x}-F_{m, x}^{\prime}=\left[f+\left(\frac{\partial v^{\prime}}{\partial x}-\frac{\partial u^{\prime}}{\partial y}\right)\right] V_{s}-W_{s} \frac{\partial u^{\prime}}{\partial z}-\frac{\partial J}{\partial x}+F_{d, x}^{\prime}, \\
& \frac{\partial v^{\prime}}{\partial t}+u^{\prime} \frac{\partial v^{\prime}}{\partial x}+v^{\prime} \frac{\partial v^{\prime}}{\partial y}+w^{\prime} \frac{\partial v^{\prime}}{\partial z}+f u^{\prime}+\frac{1}{\rho} \frac{\partial p^{H}}{\partial y}-F_{m, y}^{\prime}=-\left[f+\left(\frac{\partial v^{\prime}}{\partial x}-\frac{\partial u^{\prime}}{\partial y}\right)\right] U_{s}-W_{s} \frac{\partial v^{\prime}}{\partial z}-\frac{\partial J}{\partial y}+F_{d, y}^{\prime}
\end{aligned}
$$

où $F^{\prime}{ }_{m}$ et $F^{\prime}{ }_{d}$ représentent le mélange et la source de quantité de mouvement venant de la dissipation des vagues, respectivement.

Le terme $J$ représente une pression induite par les vagues. Dans le cas d'ondes linéaires monochromatiques avec une variance de l'élévation de surface $E, J=(g k E) /[\sinh (2 k D)]$. Les autres notations sont classiques, sauf peut-être $W_{s}$, qui est la composante verticale de la dérive de Stokes, définie précisément par ARDHUIN et al. (2008a). Par ailleurs (1) et (2) doivent être combinées à la conservation de la masse (3) et les conditions aux limites adéquates au fond et en surface (4) doivent être appliquées :

$\frac{\partial u^{\prime}}{\partial x}+\frac{\partial v^{\prime}}{\partial y}+\frac{\partial w^{\prime}}{\partial z}=0$ partout

et $\frac{\partial \eta^{\prime}}{\partial t}+\left(u^{\prime}+U_{S}\right) \frac{\partial \eta^{\prime}}{\partial x}+\left(v^{\prime}+V_{S}\right) \frac{\partial \eta^{\prime}}{\partial y}=w^{\prime}+W_{s}$ en surface

La particularité des équations (1) et (2) par rapport à ce que résout classiquement un modèle de circulation océanique, est la différence entre les vitesses d'advection des traceurs qui reste $U$, et la vitesse d'advection de la quantité de mouvement horizontale $u^{\prime}$.

Une autre possibilité, en théorie, est la résolution de l'équation d'évolution de $U$. En transformant la coordonnée verticale $z$ tel que l'on ait : $z=s(x, \zeta, t)=\eta^{\prime}+\zeta D+\tilde{s}$ avec $\zeta$ variant de -1 à 0 , ceci permettant de prendre en compte la perturbation de la position verticale des particules induite par les vagues, on obtient le modèle suivant (MELLOR, 2003 ; ARDHUIN et al., 2008b), (avec la profondeur moyenne $D=h+\eta^{\prime}$ ) :

$\frac{\partial U}{\partial t}+U \frac{\partial U}{\partial x}+\frac{\Omega}{D} \frac{\partial U}{\partial \zeta}=\frac{F}{D}$

où $\Omega$ est la vitesse verticale en coordonnée $\zeta$ et $F / D$ est l'accélération résultant la somme des forces :

- gradient de pression hydrostatique ; 
- divergence des flux de quantité de mouvement horizontaux et verticaux associés aux vagues (tensions de radiation);

- mélange.

Les expressions complètes de $F$ sont données dans les deux articles pré-cités. Nous noterons seulement ici que la divergence verticale des tensions de radiation verticales doit être estimée au même ordre que la divergence horizontale. Or, dans le cas d'un fond en pente, cela demande une solution du mouvement orbital induit par les vagues au premier ordre en pente du fond, et il n'y a pas de solution analytique de ce type. La mise en oeuvre de ce type de modèle demande donc une modélisation complexe des vagues, hors de portée pour les applications pratiques (ARDHUIN et al., 2008b). MELLOR (2003) n'a pas prêté attention à ce problème de cohérence dans l'estimation des différents termes, et il a utilisé la théorie d'Airy, pour des vagues linéaires sur fond plat, pour estimer tous les termes de forçage. Cette approche incohérente du forçage aboutit à des erreurs importantes, comme nous allons le voir. Ainsi, nous avons étudié un cas simple de la propagation de vagues sur une bosse lisse dans la direction $x$ et invariante dans la direction $y$ (cf. figures 4 et 5), en absence de frottement au fond et de dissipation. La houle se propage sur la bathymétrie avec une incidence normale et la rétroaction des courants sur les vagues n'est pas prise en compte. La solution stationnaire de (1) donne une vitesse Lagrangienne de l'ordre de $2 \mathrm{~cm} / \mathrm{s}$ pour une profondeur variant de 4 à $6 \mathrm{~m}$, un période de $5,6 \mathrm{~s}$ et une amplitude des vagues de $36 \mathrm{~cm}$.

Par contre la résolution numérique de (5) avec notre modèle couplé (dans ce cas fonctionnant seulement en forcé) utilisant l'expression du forçage par les vagues donnée par MELLOR (2003), aboutit à des vitesses beaucoup plus fortes, de l'ordre de $20 \mathrm{~cm} / \mathrm{s}$, et une solution faiblement non-stationnaire qui, localement (pour $x=200 \mathrm{~m}$ et $x=600 \mathrm{~m}$ ) a presque convergé au bout de 15 minutes de temps simulé. Toutefois, l'advection des régions de fortes vitesses finit par entraîner des instabilités numériques lorsque la région de vitesse positive s'étend et rejoint, au milieu du domaine de calcul en $x=380 \mathrm{~m}$, la région de vitesse négative.

Par ailleurs, lorsque le fond est modifié en l'étirant dans la direction $x$ d'un facteur $1 / \alpha$, les vitesses obtenues pour une profondeur fixe au temps $\mathrm{t}=\mathrm{t}_{0} / \alpha^{2}$ ne dépendent pas de la pente maximale du fond car l'équation (5) est en similitude de Froude puisque $F(x, t)$ n'est fonction que du champ de vagues à $x$ et $t$. En d'autres termes, les très fortes erreurs de vitesse subsistent sur un plateau continental en pente très douce. Seul le mélange vertical et la tension sur le fond peuvent faire varier la vitesse. Dans le cas présent un mélange vertical assez fort (viscosité turbulente constante à $2,8 \times 10^{-3} \mathrm{~m}^{2} \mathrm{~s}^{-1}$ ) a été testé et permet de réduire la vitesse en surface aux environs $12 \mathrm{~cm} / \mathrm{s}$. 


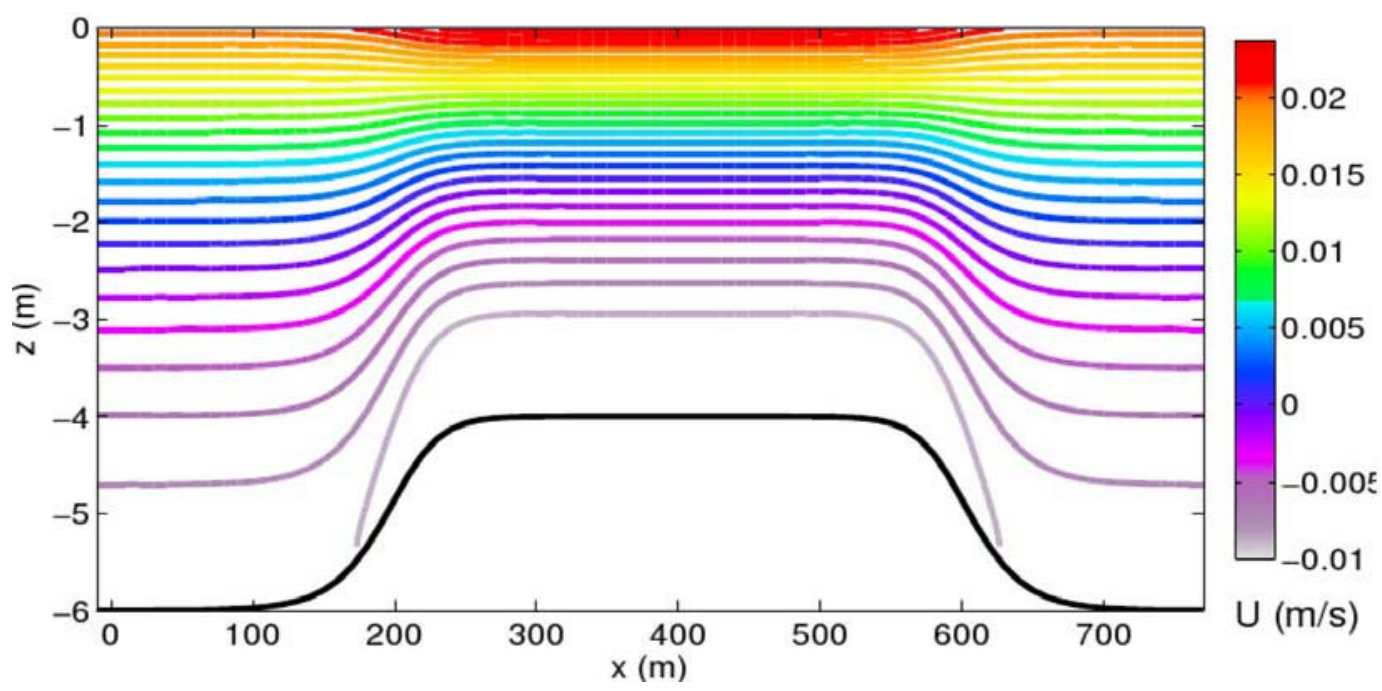

Figure 4. Cas de vagues d'amplitude $0.36 \mathrm{~m}$ et de période $5.6 \mathrm{~s}$ sur un fond en pente douce - Vitesse horizontale stationnaire obtenue en ajoutant la dérive de Stokes et la vitesse quasi-Eulérienne, solution du 2nd ordre de l'équation de Laplace.

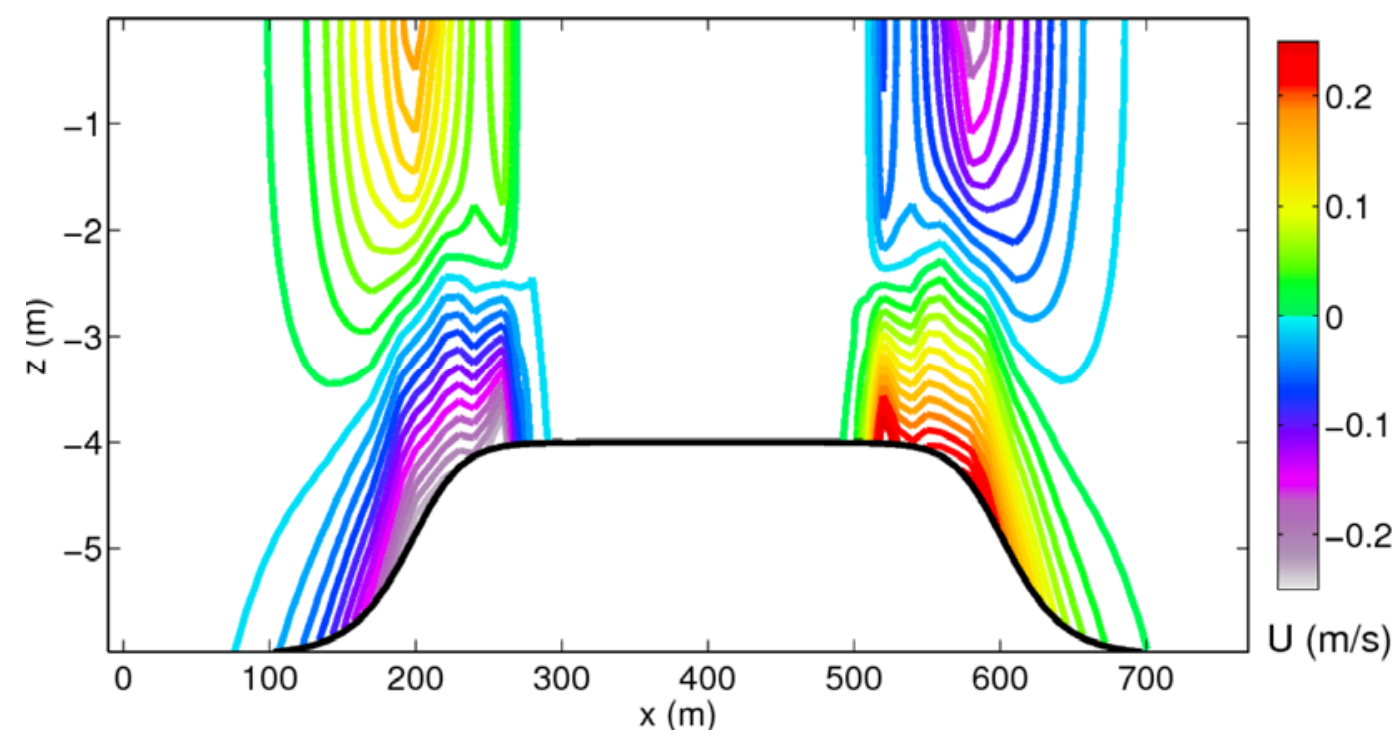

Figure 5. Cas de vagues d'amplitude $0.36 \mathrm{~m}$ et de période $5.6 \mathrm{~s}$ sur un fond en pente douce - Vitesse horizontale après 15 minutes d'intégration obtenue avec le modèle couplé résolvant les équations erronées de MELLOR (2003).

\section{Perspectives}

L'implémentation des équations (1) et (2) dans le code couplé MARS3D-WWATCH est en cours de réalisation afin de pouvoir à terme s'intéresser à des problèmes tridimensionnels réalistes. 


\section{Remerciements}

A.C. BENNIS remercie l'INSU pour son financement post-doctoral obtenu dans le cadre de l'ANR EPIGRAM. F. ARDHUIN est financé par le Conseil Européen de la Recherche (projet IOWAGA) et l'U.S. Office of Naval Research.

\section{Références bibliographiques}

ARDHUIN F., MARIE L., RASCLE N., FORGET P., ROLAND A. (2009). Obseravtion and estimation of Lagrangian, Stokes and Eulerian currents induced by wind and waves at the sea surface. J. Phys. Oceanogr., 39, pp 2820-2838. doi:10.1175/2009JPO4169.1

ARDHUIN F. (2005). Etat de la mer et dynamique de l'océan superficiel. Habilitation à diriger des recherches, Université de Bretagne Occidentale, $318 \mathrm{p}$.

ARDHUIN F., RASCLE N., BELIBASSAKIS K.A. (2008a). Explicit wave-averaged primitive equations using a Generalized Lagrangian Mean. Ocean Modelling, 20, pp 235-264.

ARDHUIN F., JENKINS A.D., BELIBASSAKIS K. (2008b). Commentary on 'the three-dimensional current and surface wave equations' by George Mellor. J. Phys. Oceanogr., 38, pp 1340-1349. doi:10.1175/2007JPO3670.1

BUIS S., PIACENTINI A., DECLAT D. (2008). PALM : A computational Framework for assembling High performance computing applications. Concurrency Computat.: Pract. Exper., 18, pp 247-262.

LAZURE P., DUMAS F. (2008). An external-internal mode coupling for $3 d$ hydrodynamical model for applications at régional scale (MARS). Adv. Water Resources, 31, pp 233-250. doi:10.1016/j.advwatres.2007.06.010

LENTZ S.J., HOWD M.F., FREDERICKS J., HATHAWAY K. (2008). Observations and a Model of undertow over the Inner Continental Shelf. J. Phys. Oceanogr., 38, pp 2341-2357. doi:10.1175/2008JPO3986.1

LONGUET-HIGGINS M.S. (1970a). Longshore Currents Generated by obliquely Incident Sea Waves, 1. J. Geophys. Res., 75, pp 6778-6789. doi:10.1029/JC075i033p06778 LONGUET-HIGGINS M.S. (1970b). Longshore Currents Generated by obliquely Incident Sea Waves, 2. J. Geophys. Res., 75, pp 6790-6801. doi:10.1029/JC075i033p06790 MELLOR G.L. (2003). The Three-Dimensional Current and Surface Wave Equations. J. Phys. Oceanogr., 33, pp 1978-1989. doi:10.1175/1520-0485(2003)033<1978:TTCASW>2.0.CO;2 TOLMAN H.L. (2008). A mosaic approach to wind wave modeling. Ocean Modelling, 25, pp 35-47. doi:10.1016/j.ocemod.2008.06.005

SVENDSEN I.A., PETREVU U. (1994). Nearshore mixing and dispersion. Proc. Roy. Soc. Lond. A, 445, pp 561-576. doi:10.1098/rspa.1994.0078

THORNTON E.B., GUZA R.T. (1986). Surf Zone Longshore Currents and Random Waves: Field Data and Models. J. Phys. Oceanogr., 16, pp 1165-1178. doi:10.1175/1520-0485(1986)016<1165:SZLCAR>2.0.CO;2 
Thème 1 - Hydrodynamique côtière

WU C.S., THORNTON E.B., GUZA R. (1985). Waves and longshore currents: Comparison of a numerical model with field data. J. Geophys. Res., 90, pp 4951-4958. doi:10.1029/JC090iC03p04951 